\title{
C4ISR FOR ADVANCED DEFENSE CAPABILITIES AND SECURITY
}

Or more than a decade we accept as ground truth the thesis that advanced
communications and information technologies, if wisely implemented, are a force multiplier. They allow the warrior to see better, to have a better orientation, to make adequate decisions faster, to hit more precisely, to access the decisions of his action more accurately. With the unfolding of the information revolution and the end of the Cold war, the information technology may become more than a force multiplier and to turn into a vehicle for promotion of regional and sub-regional security arrangements. This is particularly true after the terrorist attacks on September 11th, with the understanding of the need for comprehensive interagency and international cooperation.

No single agency may deal effectively with the new security challenges. Practically, not even single country or international organization can do that. A complex threat needs a complex response. Political, diplomatic, economic and financial, law enforcement, military and informational means of a number of actors have to be integrated in a seamless manner.

This is not a simple task even under normal circumstances. But when you search for solutions under severe resource constraints, lack of market experience and a variety of cultures of former enemies, the challenge is worth even for the most adventurous. Nevertheless, we believe that it is possible to find solutions through evolutionary development of cooperative crisis management capabilities - capabilities to collect and share information, capabilities for cooperative decision making, capabilities to control cooperative response, and capabilities to train and learn together.

This issue of Information \& Security looks for ideas how to implement advanced command and control, communications, computers, intelligence, surveillance and reconnaissance (C4ISR) technologies and systems to build and sustain cooperative crisis management capabilities in the region of South East Europe. The articles are based mostly on the experience of Bulgaria in developing defense capabilities 
through advanced C4ISR. However, they provide a glimpse at the potential of C4ISR in interagency cooperation and cooperation among the countries in the region.

Of particular importance is the approach to the acquisition of C4ISR systems when there is no obvious leader in the region, budgets are limited, technologies come from outside, and the pace of their development often exceeds the pace of political and expert coordination. In transition periods, it is a challenge to provide effectiveness of defense acquisition even for a single country. Joint procurement is not easy for countries that have been cooperating politically and economically for decades. Nevertheless, we believe that there is a great potential for joint procurement initiatives in South East Europe, and to start with procurement of C4ISR systems is the obvious choice.

The political framework is there. The United Nations and OSCE support regional security arrangements. The willingness of Western Europe and North America to sponsor the process, mostly through the Stability Pact for South East Europe, is proven. Success of SEE Defense Ministerial Process (SEDM) in the framework of Partnership for Peace/EuroAtlantic Partnership Council is great contribution to the regional security and stability. The countries in the region have the talent in labor, research, education, etc. The attraction for the business is there with the emergent market for C4ISR systems, and for advanced information and communications technologies in general.

If this volume of Information \& Security provides even tiniest acceleration to the process of regional security cooperation, it will meet our bravest expectations.

Information \& Security 